\title{
Successful Development of Viable Blastocysts From Enhanced Green Fluorescent Protein Transgene-Microinjected Mouse Embryos: Comparison of Culture Media
}

\author{
VIKRAM DEVGAN AND POLANI B. SESHAGIRI* \\ Department of Molecular Reproduction, Development and Genetics, Indian Institute of Science, Bangalore, India
}

\begin{abstract}
To improve efficiency of transgenesis, we compared M16 and CZB embryo culture media, supporting development to blastocysts of $\mathrm{FVB} /$ $\mathrm{N}$ mouse pronuclear-eggs, microinjected with enhanced green fluorescent protein (EGFP) transgene. When EGFP-injected-eggs were cultured (120 hr), blastocyst development was significantly $(P<0.03)$ higher in M16 medium $(72.5 \pm 2.4 \%)$ than that in CZB $(13.2 \pm 4.3 \%)$ or CZBG (CZB with $5.6 \mathrm{mM}$ glucose at $48 \mathrm{hr}$ culture) $(62.1 \pm 3.7 \%)$ media. Blastocyst development of noninjected embryos was higher in M16 $(92.0 \pm 2.6 \%)$ and CZBG $(83.9 \pm 3.9 \%)$ media than in CZB $(31.9 \pm 2.8 \%)$ medium $(P<0.0001)$. However, percentages of morulae at $72 \mathrm{hr}$ were comparable in all treatments. Developed blastocysts were better in M16 than in CZB or CZBG media. Consistent with this, mean cell number per blastocyst, developed from injected embryos, was significantly $(P<0.002)$ higher in M16 medium (79.6), than those in CZB (31.3) or CZBG media (60.7); similar with noninjected embryos. Cell allocation to trophectoderm (TE) and inner cell mass (ICM), i.e., TE:ICM ratio, for injected blastocysts in M16 (3.0) was less than $(P<0.05)$ those in CZB (4.2) and CZBG (4.4) media; similar with noninjected blastocysts. Moreover, blastocysts, developed in M16 and CZBG media, hatched, attached, and exhibited trophoblast outgrowth; 18\% of them showed EGFP-expression. Importantly, blastocysts from M16 medium produced live transgenic "green" pups (11\%) following embryo transfer. Taken together, our results indicate that supplementation of glucose, at $48 \mathrm{hr}$ of culture (CZBG), is required for morula to blastocyst transition; M16 medium, containing glucose from the beginning of culture, is superior to CZB or CZBG for supporting development of biologically viable blastocysts from EGFPtransgene-injected mouse embryos. Mol. Reprod. Dev. 65: 269-277, 2003. () 2003 Wiley-Liss, Inc.
\end{abstract}

Key Words: green fluorescent protein; culture media; mouse embryo; blastocyst development; transgenesis

\section{INTRODUCTION}

Transgenic animals are important for scientific and pharmaceutical purposes. Pronuclear microinjection is the most widely used method for mouse transgenesis. Its limitation, however, is the low efficiency. One of the major reasons for this is the substantial loss of viability of injected embryos during in vitro culture or in vivo, following their transfer to the oviducts of pseudopregnant recipients. Since, preimplantation-stage embryos are most vulnerable to mortality, both in vivo and in vitro, it is desirable to culture transgene-injectedpronucleate-eggs to blastocysts and then select developmentally competent and potentially viable (transgenic) blastocysts for uterine transfer (Takada et al., 1997; Kato et al., 1999; Keiser et al., 2001). This approach i.e., embryo-culture-based transgenesis (ECBT), however, has not been extensively practiced.

Although the conditions for short-term culture of injected-pronuclear-eggs are standardized, a few desirable modifications are required, considering the epigenetic regulation of embryo development by energy substrates and amino acids (Bavister, 1995; Gardner, 1998). The embryonic requirement and preferences of these molecules change with advancing development (Gardner, 1998). In the mouse, only pyruvate (or oxaloacetate) can support development of 1-cell embryos, lactate can be utilized from the 2-cell stage, while glucose can serve as the sole substrate from the 8-cell stage (Biggers et al., 1967). Embryonic-stage-dependent requirement for glucose during early mouse development has been studied (Chatot et al., 1994; Martin

Data contained in this article was presented at the 35th Annual Meeting of the Society for the Study of Reproduction, held in Baltimore, MD and the abstract was published in: Biol Reprod 66(Suppl 1):155 (abstract no. 141).

Grant sponsor: Department of Biotechnology, New Delhi.

*Correspondence to: Prof. Polani B. Seshagiri, Department of Molecular Reproduction, Development and Genetics, Indian Institute of Science, Bangalore 560012, India.

E-mail: polani@mrdg.iisc.ernet.in

Received 28 November 2002; Accepted 30 January 2003

Published online in Wiley InterScience (www.interscience.wiley.com) DOI $10.1002 / \mathrm{mrd} .10306$ 
and Leese, 1995; Leppens-Luisier and Sakkas, 1997). Careful selection of energy substrates, therefore, is critical for successful development of embryos. In this regard, there is a need to assess routinely used culture media such as M16 (Whittingham, 1971) and CZB (Chatot et al., 1989) for supporting development of transgene-injected-mouse embryos. The CZB medium differs from the M16 medium, in terms of containing high lactate:pyruvate ratio and $1 \mathrm{mM}$ glutamine and is devoid of glucose (Chatot et al., 1989). Of relevance, here, is the observation that glucose-free medium facilitates in vitro development of preimplantation embryos in a variety of species such as hamster (Schini and Bavister, 1988; Seshagiri and Bavister, 1989, 1991; Ain and Seshagiri, 1997), cow (Takahashi and First, 1992), sheep (Thompson et al., 1992), pig (Petters et al., 1990), including humans (Conaghan et al., 1993). However, it is not clear whether there is a critical and stage-dependent need for glucose in the culture media that supports development of microinjected-embryos to blastocysts in the mouse.

Using conventional technology, we earlier generated enhanced green fluorescent protein (EGFP) transgenic mice with variable phenotypes in term of transgene expression (Devgan et al., 2003). Using embryo-culturebased transgenesis, Kato et al. (1999) have produced EGFP-transgenic mice by selective transfer of EGFPexpressing blastocysts. Because EGFP-expression (fluorescence) can be monitored noninvasively in living cells and it does not require a substrate or a pretreatment for detection, it has become a suitable genetic reporter system for monitoring transgene expression during embryogenesis. To the best of our knowledge, however, there has been no report on the in vitro development through hatched and peri-attachment blastocyst stages of microinjected-pronuclear-stage eggs in the mouse and, whether or not there is a need for supplementation of glucose in culture media. In this study, we investigated relative abilities of M16 and CZB (without or with glucose) media to support in vitro development of EGFP-microinjected pronuclear embryos through peri-attachment blastocyst stages and assessed their quality and biological viability in the mouse.

\section{MATERIALS AND METHODS}

\section{Animals}

Four to six week old FVB/N and C57BL/6 mice, maintained on a $14 \mathrm{~L}: 10 \mathrm{D}$ lighting schedule (lights on at 06:00 hr) and normal temperature $\left(24-26^{\circ} \mathrm{C}\right)$, from our transgenic mouse facility were used for the present study. Procedures for handling and experimentation followed the Guidelines on the Use of Animals in Scientific Research (INSA, New Delhi, 1992).

\section{Construction of EGFP Vector}

We used EGFP, a red shifted and humanized GFP variant, as the transgene as before (Devgan et al., 2003). The cDNA encoding EGFP was amplified by polymerase chain reaction (PCR) using pEGFP1 as a template (Clontech Co. Ltd., Palo Alto, CA). The EcoRI sites, engineered in primers, were used to introduce the amplified EGFP cDNA into pCAGGS expression vector containing the cytomegalovirus (CMV-IE) enhancer, chicken- $\beta$-actin (C $\beta A$ ) promoter, $\beta$-actin intron and rabbit $\beta$-globin poly-A signal. The entire insert (C $\beta A$ EGFP), which included the promoter and coding sequences, was excised with BamHI and SalI, electrophoresed and gel purified using Qiagen gel elution kit (Qiagen, Chatsworth, CA). The resulting $3.2 \mathrm{~kb}$ transgene was used for pronuclear egg-microinjection (Devgan et al., 2003).

\section{Microinjection, Embryo Culture and Embryo Transfer}

FVB/N females were superovulated by i.p. injection of 5 i.u. pregnant mare's serum gonadotropin (Sigma Chemical Company, St. Louis, MO), followed $52 \mathrm{hr}$ later by 5 i.u. human chorionic gonadotropin (hCG; Sigma) and mated with proven stud males. Pronuclear-stage embryos were collected from excised oviducts at $20 \mathrm{hr}$ post-hCG in M2 medium (Sigma). The purified transgene (EGFP; $5 \mathrm{ng} / \mu \mathrm{l}$ ) was microinjected (Transjector 5246, Eppendorf, Barkhausenweg, Hamburg, Germany) into the male pronuclei of embryos (Devgan et al., 2002). Injected and noninjected (control) embryos from each set of experiment were cultured in $50 \mu \mathrm{l}$ drops of the M16 (Sigma) or CZB (indigenously prepared from Sigma chemicals) or CZBG (CZB with $5.6 \mathrm{mM}$ glucose, added at $48 \mathrm{hr}$ of culture period) media. Since M16 medium contained $5.6 \mathrm{mM}$ glucose, a similar concentration of glucose was added in CZB instead of $27 \mathrm{mM}$ as used by Chatot et al. (1994). During culture, media were overlaid with $2.5 \mathrm{ml}$ of silicone oil in $35 \mathrm{~mm}$ plastic culture dishes (Greiner Labortechnik, Frickenhausen, Germany), maintained at $37^{\circ} \mathrm{C}$ with a humidified gas atmosphere of $5 \% \mathrm{CO}_{2}$ in air. Embryo development was monitored microscopically every $24 \mathrm{hr}$ until $120 \mathrm{hr}$.

Embryo transfer procedure was as described previously (Ain and Seshagiri, 1997; Devgan et al., 2002). Briefly, about 20 blastocysts (EGFP-expressing and nonexpressing), cultured in M16 medium, were unilaterally transferred to uteri of day 3 pseudopregnant recipients $(\mathrm{C} 57 \mathrm{BL} / 6)$; among these, atleast $2-3$ were green blastocysts. Transferred embryos were allowed to develop to term. At birth, their transgenic status was monitored as described below.

\section{Genotypic Analysis}

All animals, at the time of weaning, were genotyped using tail DNA sample by PCR and genomic Southern analyses as described previously (Devgan et al., 2003). Briefly, genomic DNA was isolated from tail tips of 46 week-old mice according to standard procedure (Hogan et al., 1986). Approximately, $200 \mathrm{ng}$ of genomic DNA was used as a template in PCR. The amplification reaction was carried out with $1.25 \mathrm{U}$ of Taq polymerase (Promega, Madison, WI) in a mixture containing $1 \times$ Taq buffer, $200 \mu \mathrm{M}$ dNTP and $0.2 \mu \mathrm{M}$ primers. Primers used 
for EGFP were: 5' ACGTAAACGGCCACAAGTTC $3^{\prime} ; 5^{\prime}$ TGTTCTGCTGGTAGTGGTCG $3^{\prime}$ and for glyceraldehydes-3-phosphate dehydrogenase (GAPDH) were: $5^{\prime}$ GAGTATGTTGTGGAGTCTACTGGCG 3'; $5^{\prime}$ TGTCATTGAGAGCAATGCCAGCC $3{ }^{\prime}$. The PCR mixture was heated at $95^{\circ} \mathrm{C}$ for $10 \mathrm{~min}$, then amplified for 30 cycles, denatured at $94^{\circ} \mathrm{C}$ for $1 \mathrm{~min}$, annealed at $60^{\circ}$ (EGFP) or $65^{\circ}$ (GAPDH) for $1 \mathrm{~min}$ and extensions were carried out at $72^{\circ} \mathrm{C}$ for $1 \mathrm{~min}$, followed by $5 \mathrm{~min}$ of extension at $72^{\circ} \mathrm{C}$. PCR products $(5 \mu \mathrm{l})$ were then analyzed by ethidium bromide-stained 1.5\% agarose gel electrophoresis.

For genomic Southern analysis, genomic DNA $(10 \mu \mathrm{g})$ was digested with $E c o$ RI, subjected to $0.8 \%$ agarose gel electrophoresis, followed by transfer of resolved DNA to Hybond- ${ }^{+}$membrane (Amersham, Buckinghamshire, UK). The EcoRI fragment, containing EGFP cDNA from pCAGGS-EGFP, was radiolabeled by random priming (Random Prime Labeling Kit, Bangalore Genei, Bangalore, India) with $\left[\alpha_{-}{ }^{32} \mathrm{P}\right] \mathrm{dCTP}$ and used as a probe. Hybridization was carried out at $65^{\circ}$ for $16 \mathrm{hr}$. The membrane was washed twice with $2 \times \operatorname{SSC}(1 \times=0.15 \mathrm{M}$ $\mathrm{NaCl}, 0.015 \mathrm{M}$ sodium citrate, $\mathrm{pH} 7.0$ ), containing $0.1 \%$ $\mathrm{SDS}$ at room temperature, then once with $0.2 \times \mathrm{SSC}$ containing $0.1 \%$ SDS once at room temperature and once at $65^{\circ} \mathrm{C}$. Finally, the membrane was washed with $0.1 \times$ SSC containing $0.5 \% \mathrm{SDS}$ at $69^{\circ} \mathrm{C}$ and analyzed using BAS 1800 phosphoimager (Fuji Photo Film, Shinjukuku, Tokyo, Japan).

\section{Differential Staining of Blastocysts}

Allocation of cells to trophectoderm (TE) and inner cell mass (ICM) of cultured blastocysts was determined following the procedure of Handyside and Hunter (1984) with modification (Mishra and Seshagiri, 1998). Briefly, blastocysts were treated with $0.4 \%$ pronase to digest zona pellucida. Azonal blastocysts were washed in M2 medium and then incubated in 1:5 diluted, heat inactivated rabbit anti-mouse spleenocyte antiserum (raised in our laboratory) for $10 \mathrm{~min}$ at $37^{\circ} \mathrm{C}$. Blastocysts were then washed and transferred to 1:5 diluted guinea pig serum containing $15 \mu \mathrm{g} / \mathrm{ml}$ of propidium iodide at $37^{\circ} \mathrm{C}$ for $5-6$ min till all TE cells took up the stain as visualized under fluorescence inverted microscopy (IX70, Olympus, Tokyo, Japan). Blastocysts were then rapidly rinsed in M2 medium and transferred to a drop of counterstain-cocktail $(750 \mu \mathrm{l}$ of $2.3 \%$ sodium citrate, $250 \mu \mathrm{l}$ of $96 \%$ ethanol and $30 \mu \mathrm{l}$ of $1 \mathrm{mg} / \mathrm{ml}$ Hoechst stock solution) for $3 \mathrm{~min}$ at $37^{\circ} \mathrm{C}$. Stained blastocysts were mounted with glycerol on siliconized glass slides and observed under the microscope as above. Under UV illumination, TE cells appear pink while ICM cells appear blue. A sum of TE and ICM cells per blastocysts was considered for determining mean cell number (MCN) per blastocyst.

\section{Blastocyst Attachment and Morphometric Analysis}

Azonal blastocysts were cultured in M16 or CZBG media containing $10 \%$ bovine fetal serum (BFS; Hyclone Laboratories Inc., Logan, Utah) in dishes precoated with neat BFS as described elsewhere (Mishra and Seshagiri, 1998). Blastocysts, that did not move when the medium was gently swirled, were considered as attached. Trophoblast (TB) outgrowth was observed as the appearance of a monolayer of cells around the embryo when monitored at every $24 \mathrm{hr}$ up to $168 \mathrm{hr}$ under the inverted Olympus microscope (IX-70) using phase contrast objectives. Photographs were taken using a 100 ASA Kodak film and then subjected to a computerized scanning (HP-Scanner, 300 dpi) to determine the area of the TB outgrowth using an image analysis software programme, Image Pro Plus (Media Cybernetics, Silver Spring, MD).

\section{Monitoring of Embryonic/Neonatal EGFP Fluorescence}

The protein expression of EGFP in microinjectedembryos throughout pre/peri-implantation development or live pups was examined under fluorescence inverted microscope (IX-70, Olympus) or stereozoom microscope (SZX-RFL2, Olympus) with GFP filter set. Fluorescent pictures were photographed using a 400 ASA Kodak film with the photomicrographic unit (PM-30, Olympus) attached to the microscope.

\section{Statistical Analysis}

All experiments were performed for a minimum of five times. Results are expressed as mean \pm SEM. Percentage values were subjected to arcsin transformation before statistical analysis. Comparisons between groups were made by the unpaired Student $t$-test (PRISM Graph Pad version 2; Graph Pad Software, San Diego, CA). Value sets showing $P<0.05$ were considered as statistically significant.

\section{RESULT \\ Development of Blastocysts From Microinjected-Pronuclear-Stage Eggs}

The CBA-EGFP transgene was microinjected into 566 pronucleate-eggs. Of these, $470(83 \%)$ survived injection and were cultured in M16, CZB, and CZBG media. The data of this is shown in Table 1 . After $72 \mathrm{hr}$ of culture, $81.3 \pm 4.1 \%, 72.6 \pm 3.8 \%$, and $78.9 \pm 3.1 \%$ embryos developed to morulae in M16, CZB, and CZBG media, respectively. However, after $120 \mathrm{hr}$ of culture, $72.5 \pm 2.4 \%$ embryos developed to blastocysts in M16 medium, whereas only $13.2 \pm 4.3 \%$ and $62.1 \pm 3.7 \%$ embryos developed to blastocysts in CZB and CZBG media, respectively. While the percentages of morula development were comparable in all three media, the percentage of blastocyst development of injectedembryos in M16 medium was significantly higher $(P<0.03 ; \mathrm{n}=10)$ than those in CZB and CZBG media (Table 1; Fig. 1).

Similarly, when noninjected (control) pronuclearstage embryos were cultured in M16, CZB, and CZBG media (Table 1), $95.5 \pm 2.4 \%, 90.7 \pm 3.1 \%$, and $88.6 \pm$ $2.8 \%$ embryos developed to morulae, respectively. However, $92.0 \pm 2.6 \%$ and $83.9 \pm 3.9 \%$ embryos developed to 
TABLE 1. Development of Morulae or Blastocysts Following EGFP-Transgene Microinjection Into Pronuclear-Stage Mouse Embryos

\begin{tabular}{|c|c|c|c|c|}
\hline \multirow[b]{2}{*}{ Treatment } & \multirow[b]{2}{*}{ Category } & \multirow{2}{*}{$\begin{array}{l}\text { No. of embryos } \\
\text { cultured }\end{array}$} & \multicolumn{2}{|c|}{ Development $^{a}(\%)$} \\
\hline & & & Morula & Blastocyst \\
\hline \multirow[t]{2}{*}{ M16 } & Noninjected & 201 & $191\left(95.5 \pm 2.4^{\mathrm{b}}\right)$ & $186\left(92.0 \pm 2.6^{\mathrm{e}, \mathrm{f}}\right)$ \\
\hline & Injected & 159 & $134\left(81.3 \pm 4.1^{b}\right)$ & $118\left(72.5 \pm 2.4^{\mathrm{e}, \mathrm{g}, \mathrm{h}}\right)$ \\
\hline \multirow[t]{2}{*}{$\mathrm{CZB}$} & Noninjected & 173 & $155\left(90.7 \pm 3.1^{c}\right)$ & $56\left(31.9 \pm 2.8^{\mathrm{f}, \mathrm{i}, \mathrm{j}}\right)$ \\
\hline & Injected & 161 & $113\left(72.6 \pm 3.8^{\mathrm{c}}\right)$ & $23\left(13.2 \pm 4.3^{\mathrm{g}, \mathrm{i}, \mathrm{k}}\right)$ \\
\hline \multirow[t]{2}{*}{ CZBG } & Noninjected & 168 & $145\left(88.6 \pm 2.8^{\mathrm{d}}\right)$ & $137\left(83.9 \pm 3.9^{\mathrm{c}, \mathrm{j}, \mathrm{l}}\right)$ \\
\hline & Injected & 150 & $121\left(78.9 \pm 3.1^{d}\right)$ & $91\left(62.1 \pm 3.7^{\mathrm{h}, \mathrm{k}, \mathrm{l}}\right)$ \\
\hline
\end{tabular}

${ }^{a}$ Development was assessed at $72 \mathrm{hr}$ (morula) or $120 \mathrm{hr}$ (blastocyst). Values in parentheses represent mean \pm SEM, from ten replicate experiments.

Values with identical superscripts differ significantly. b: $P<0.01$; c, i, l: $P<0.002$; d, h: $P<0.05$; e, f, g, j, k: $P<0.03$.

blastocysts in M16 and CZBG media, respectively, while only $31.9 \pm 2.8 \%$ embryos developed to blastocysts in CZB medium (Table 1; Fig. 1). While the percentages of morula development were comparable in all three media, the percentages of blastocyst development of noninjected pronuclear-stage embryos in M16 and CZBG were significantly $(P<0.0001)$ higher than that in the CZB medium. Moreover, percentages of morula $(P<0.05)$ and blastocyst $(P<0.002)$ development of noninjected embryos were significantly higher than those of EGFP-injected embryos in all three media. Figure 2 shows a representative example of photomicrographic pictures of in vitro developed blastocysts from noninjected and EGFP-injected embryos in M16, CZB, and CZBG media. Morphologically, blastocysts developed in M16 medium were the best, in terms of visual assessment and the extent of blastocoel expansion, followed by CZBG and CZB, regardless of whether or not the eggs were injected.



Fig. 1. Development of blastocysts from noninjected eggs $(\square$ or EGFP-microinjected-pronuclear-stage mouse eggs (W) after $120 \mathrm{hr}$ of culture. Results represent mean \pm SEM of ten replicate experiments, each with approximately 15 embryos per treatment. Values with identical superscripts differ significantly. $P$ values: a, b, d, e $<0.0001$; $\mathrm{c}<0.03$.
To compare relative abilities of commercial vs. indigenously prepared M16 medium, both noninjected and EGFP-injected embryos were cultured in the two media and the blastocyst developmental status was assessed. When cultured in indigenously prepared M16 medium, $93.5 \pm 3.2 \%(95 / 101)$ and $71.3 \pm 2.1 \%(61 / 85)$ of noninjected and EGFP-injected pronuclear-stage eggs, respectively, developed to blastocysts. While blastocyst development, achieved in commercial medium, for noninjected and injected pronuclear-stage embryos were $92.0 \pm 2.6 \%$ and $72.5 \pm 2.4 \%$, respectively (Table 1 ). Moreover, the percentages of blastocyst development in indigenously prepared M16 medium, were quite similar to those in commercial M16 medium (Table 1; $P>0.5 ; \mathrm{n}=5$ ).

\section{Assessment of Quality of Developed Blastocysts}

Qualities of in vitro developed blastocysts were judged by determining the MCN per blastocyst. The data is shown in Table 2. The MCN of blastocysts, developed from injected embryos, cultured in the M16 medium $(79.6 \pm 4.7)$ was significantly $(P<0.002)$ higher than those cultured in CZB ( $31.3 \pm 1.7)$ and CZBG $(60.7 \pm 2.2$; Fig. 3) media. Similarly, MCN of blastocysts, developed from noninjected embryos, cultured in the M16 (92.7 \pm 2.4) were significantly $(P<0.0001)$ higher than those cultured in CZB $(33.9 \pm 1.1)$ and CZBG $(72.4 \pm 3.5$; Fig. 3) media. Furthermore, blastocysts developed from noninjected embryos had significantly $(P<0.02)$ higher MCN compared to those developed from injected embryos in M16 and CZBG media (Table 2; Fig. 3), whereas MCN remained similar $(P>0.05)$ in blastocysts developed from noninjected and injected embryos in CZB medium.

The cell allocation to TE and ICM, as reflected by TE:ICM ratio, for injected embryos cultured in M16 $(3.0 \pm 0.4)$ was significantly lesser $(P<0.05)$ than those in CZB ( $4.2 \pm 0.4)$ and CZBG $(4.4 \pm 0.2)$ media (Table 2 ; Fig. 4). Similarly, for noninjected embryos, the TE:ICM ratio for M16-cultured blastocysts was lesser $(2.6 \pm 0.2)$ than those in CZB $(3.8 \pm 0.3)$ and CZBG $(4.3 \pm 0.3)$ media $(P<0.03$; Fig. 4$)$. Whereas, TE:ICM ratio of 

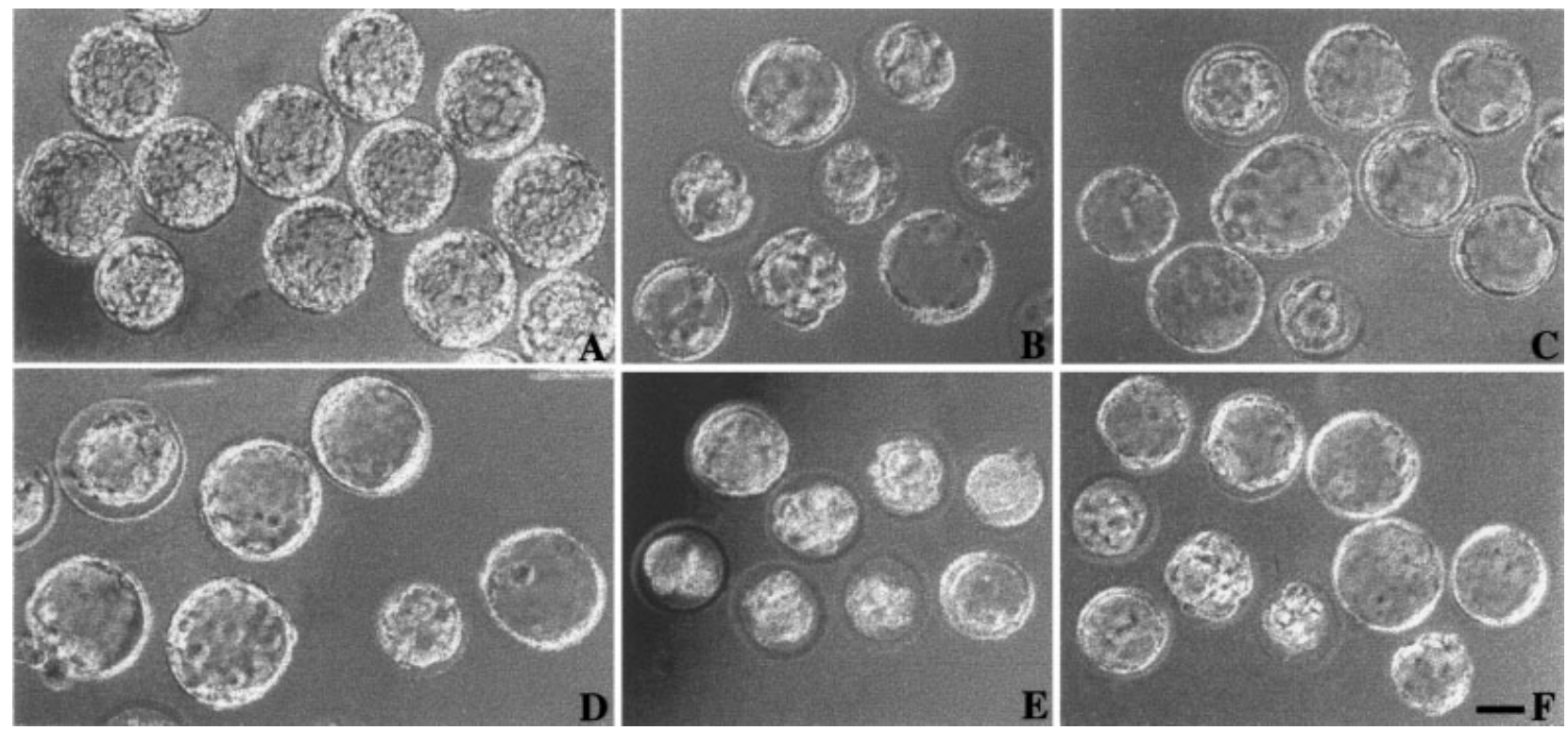

Fig. 2. Photomicrographs of in vitro developed blastocysts from noninjected (panels A-C) and EGFPinjected (D-F) pronuclear-stage mouse eggs in M16 (A, D), CZB (B, E), and CZBG (C, F) media, after $120 \mathrm{hr}$ of culture. Photographed with Olympus IX-70 microscope with Nomarski optics with $20 \times$ objective. Magnification bar shown in panel $\mathrm{F}$ corresponds to $50 \mu \mathrm{m}$ and is identical for all panels in the figure.

blastocysts developed from noninjected embryos were comparable to that of injected embryos in all the three media (Table 2; Fig. 4).

\section{Development of Blastocysts Through Peri-Attachment Stages}

The viability of blastocysts was confirmed by their ability to hatch out of the zona pellucida, attach to substratum and to exhibit trophoblast outgrowth. After $144 \mathrm{hr}$ of culture, about $91.2 \pm 2.7 \%$ (51/56), $11.7 \pm 7.3 \%$ $(2 / 15)$, and $78.3 \pm 2.2 \%(40 / 51)$ of blastocysts, developed from injected-pronuclear-stage eggs, underwent hatching in M16, CZB, and CZBG media, respectively. The rate of blastocyst hatching in M16 medium was significantly higher $(P<0.01 ; \mathrm{n}=5)$ than those in CZB and CZBG media. Hatched blastocysts, when transferred to BFS-coated dishes, attached and exhibited TB outgrowth in a time-dependent manner (Fig. 5). In the injected category, about $93 \%(14 / 15), 80 \%(12 / 15)$ of hatched blastocysts cultured in M16 and CZBG media, respectively, attached to substratum. After attachment, all embryos exhibited a continuous increase in the area of TB outgrowth, and maximal outgrowth was observed at $96 \mathrm{hr}$, i.e., $68.9 \times 10^{3} \pm 3.0 \times 10^{3} \mu \mathrm{m}^{2}$ in M16 and $65.5 \times 10^{3} \pm 3.0 \times 10^{3} \mu \mathrm{m}^{2}$ in CZBG-cultured embryos. Subsequently, by $168 \mathrm{hr}$, the extent of TB outgrowth decreased to $55.6 \times 10^{3} \pm 1.5 \times 10^{3} \mu \mathrm{m}^{2}$ and $52.0 \times 10^{3} \pm$ $1.5 \times 10^{3} \mu \mathrm{m}^{2}$ in M16 and CZBG media, respectively (Fig. 6). The area of TB outgrowth in the M16 medium was not significantly different $(P>0.05)$ from that in the CZBG medium. Moreover, all blastocysts $(47 / 47 ; 100 \%)$, developed from noninjected embryos, cultured in M16

TABLE 2. Comparison of Mean Cell Number and Cell Allocation to Trophectoderm and Inner Cell Mass in Cultured Blastocysts From NonInjected/EGFP-InjectedPronuclear-Stage Mouse Embryos

\begin{tabular}{|c|c|c|c|c|c|}
\hline \multirow[b]{2}{*}{ Treatment } & \multirow[b]{2}{*}{ Category } & \multicolumn{4}{|c|}{ Mean $\pm \operatorname{SEM}^{a}$} \\
\hline & & $\mathrm{TE}$ & ICM & Total (MCN) & TE/ICM \\
\hline \multirow[t]{2}{*}{ M16 } & Noninjected & $64.9 \pm 1.9$ & $27.9 \pm 1.6$ & $92.7 \pm 2.4^{\mathrm{b}, \mathrm{c}, \mathrm{d}}$ & $2.6 \pm 0.2^{\mathrm{j}, \mathrm{k}}$ \\
\hline & Injected & $56.9 \pm 2.9$ & $22.7 \pm 2.8$ & $79.6 \pm 4.7^{\mathrm{b}, \mathrm{e}, \mathrm{f}}$ & $3.0 \pm 0.4^{1, \mathrm{~m}}$ \\
\hline \multirow[t]{2}{*}{ CZB } & Noninjected & $26.6 \pm 1.0$ & $7.9 \pm 0.4$ & $33.9 \pm 1.1^{\mathrm{c}, \mathrm{g}}$ & $3.8 \pm 0.3^{j}$ \\
\hline & Injected & $25.3 \pm 1.6$ & $6.4 \pm 0.4$ & $31.3 \pm 1.7^{\mathrm{e}, \mathrm{h}}$ & $4.2 \pm 0.4^{1}$ \\
\hline \multirow[t]{2}{*}{ CZBG } & Noninjected & $58.3 \pm 2.5$ & $14.1 \pm 1.3$ & $72.4 \pm 3.5^{\mathrm{d}, \mathrm{g}, \mathrm{i}}$ & $4.3 \pm 0.3^{\mathrm{k}}$ \\
\hline & Injected & $50.9 \pm 1.6$ & $11.8 \pm 0.7$ & $60.7 \pm 2.2^{\mathrm{f}, \mathrm{h}, \mathrm{i}}$ & $4.4 \pm 0.2^{\mathrm{m}}$ \\
\hline
\end{tabular}

${ }^{a}$ Values shown are obtained from 15 embryos from three experiments.

Values with identical superscripts differ significantly. b, l: $P<0.05 ; \mathrm{c}, \mathrm{d}$, e, g, h, k: $P<0.0001$; f: $P<0.001 ;$ i, j, m: $P<0.01$. 


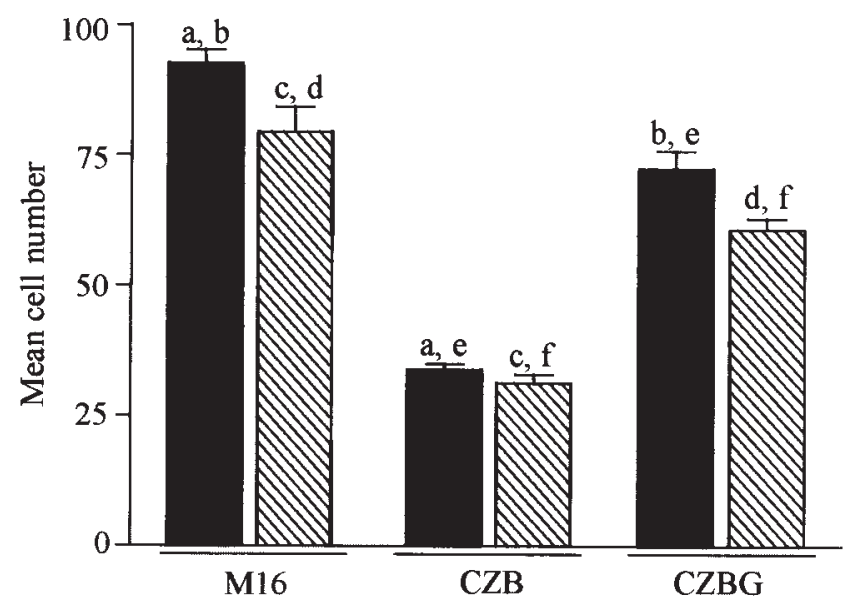

Fig. 3. Mean cell number of cultured blastocysts from uninjected and injected $(\mathbb{N})$ mouse embryos. Results represent mean \pm SEM of 15 embryos per treatment. Values with identical superscripts differ significantly. $P$ values: a, b, c, e, f $<0.0001 ; \mathrm{d}<0.002$.

medium underwent hatching, attached $(15 / 15 ; 100 \%)$ and exhibited TB proliferation with area reaching $75.5 \times 10^{3} \pm 2.1 \times 10^{3} \mu \mathrm{m}^{2}$ at $96 \mathrm{hr}$ and then decreasing to $61.8 \times 10^{3} \pm 1.8 \times 10^{3} \mu \mathrm{m}^{2}$ by $168 \mathrm{hr}$.

\section{EGFP Expression in Developed Blastocysts Through Peri-Attachment Stages}

EGFP expression was observed during the entire period of pre- and peri-implantation embryo development in vitro. About, 18\% of developed blastocysts showed green fluorescence, which persisted through the entire peri-attachment stages. EGFP expression in blastocysts was observed in undifferentiated inner cell mass as well as in TE cells (Fig. 7I). Furthermore, expanded- hatching- hatched and attached blastocysts exhibited EGFP expression (Fig. 7II). In attached blastocysts, inner stem cell as well as TB showed green fluorescence (Fig. 7III).

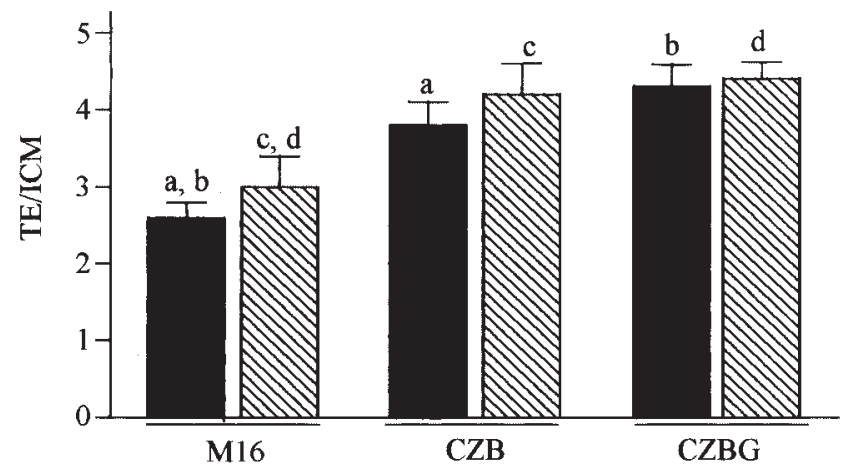

Fig. 4. Allocation of cells to trophectoderm (TE) and inner cell mass (ICM) in cultured blastocysts from noninjected $\square$ and injected $\mathbb{\mathbb { N }}$ mouse embryos. Results shown on the Y-axis are ratios of TE/ICM numbers as described in Table 2. Values represent mean \pm SEM of 15 embryos per treatment. Values with identical superscripts differ significantly. $P$ values: a, d $<0.005 ; \mathrm{b}<0.0001 ; \mathrm{c}<0.05$.

\section{Generation of Transgenic "Green” Mice}

To investigate the biological viability, 248 M16cultured-blastocysts (45 EGFP-expressing and 203 nonexpressing), developed from EGFP-injected embryos, were transferred to uteri of 12 day 3 pseudopregnant recipients. Of these, six (50\%) females became pregnant and at term, they delivered $29(25 \% ; 29 / 118)$ pups. Unfortunately, 10 pups were lost due to maternal cannibalism. Among the surviving neonatal animals, two showed ubiquitous green fluorescence on the entire body surface. Genotypic (PCR) analysis of surviving animals showed that a transgene-specific $496 \mathrm{bp}$ fragment was amplified from two animals viz., uterine transfer (UT)-8 and UT-14 (2/19; 11\%; Fig. 8A), whereas no amplification was detected with genomic DNA of other mice which also did not exhibit green fluorescence. To confirm the integration of the transgene in UT- 8 and UT-14, Southern blot analysis of EcoR I digested genomic DNA was carried out using EGFP cDNA probe. This showed hybridization signal of a predicted $732 \mathrm{bp}$ fragment from UT-8 and UT-14 (Fig. 8B). These genotypic data confirmed the transgenicity of the two (UT-8 and UT-14) founder mice. These two founder mice were fertile and produced transgenic progeny in the F1 generation as per the Mendelian pattern of genetic inheritance (data not shown).

\section{DISCUSSION}

The present study shows that M16 medium supports maximal development of high quality blastocysts from EGFP-transgene-injected or noninjected pronuclearstage mouse embryos. Blastocysts, cultured in this medium, are not only of high quality but also retain their ability to hatch, attach and exhibit extensive TB outgrowth, accompanied by EGFP protein expression. Moreover, developed blastocysts produce live transgenic "green" offspring, following uterine-embryo transfer to day 3 pseudopregnant recipients. Incidentally, this is the first report of blastocyst attachment and TB outgrowth following development of transgene-injected mouse embryos. Although embryo-culture-based transgenesis is emerging as a relatively more efficient approach (Takada et al., 1997; Kato et al., 1999; Keiser et al., 2001), only limited information is available on the assessment of developmental competence of microinjected embryos. In this regard, our data is quite significant in understanding the M16 (vs. CZBG) supported-development of microinjected embryos through peri-attachment stages and their potential to produce live offspring.

Our results show that though the injected-embryos develop to normal morulae in a glucose-free (CZB) medium, they obligatorily require glucose for cavitation and formation of high quality blastocysts. In the absence of glucose, majority of CZB-developed morulae degenerate and only $13 \%$ of them develop to blastocysts, accompanied by reduced cell numbers; of these, only $17 \%$ hatch. However, supplementation of glucose at $48 \mathrm{hr}$ of culture (CZBG), rescue embryos and $68 \%$ of 
I



II

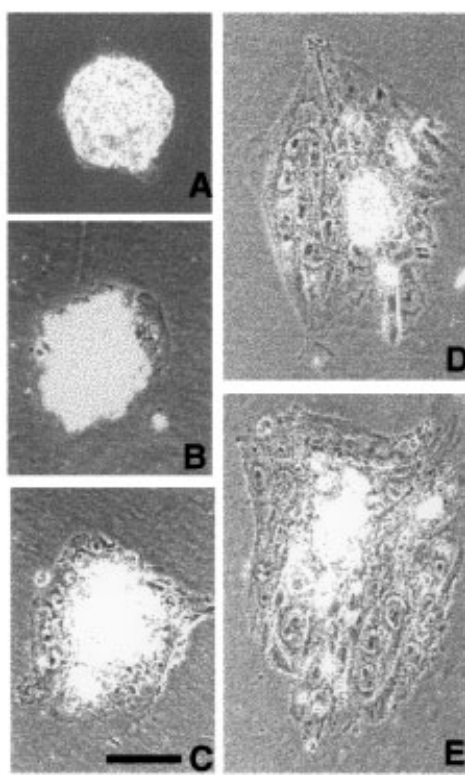

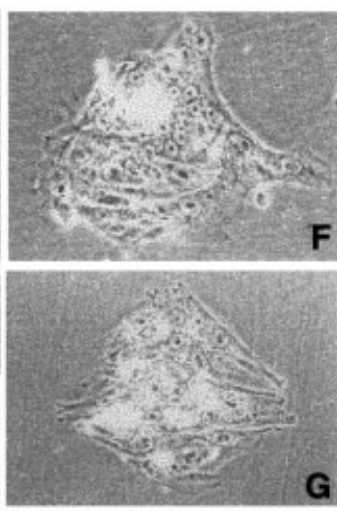

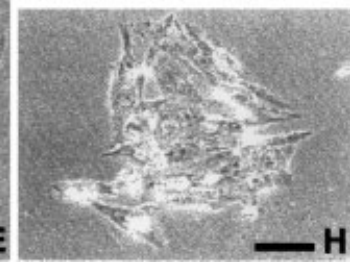

Fig. 5. In vitro development of injected embryos through peri-attachment stages in the mouse. Panels I and II show progressive changes in the morphology of attached embryos after 12 (A), 24 (B), 48 (C), 72 (D), $96(\mathbf{E}), 120(\mathbf{F}), 144(\mathbf{G})$, and $168 \mathrm{hr}(\mathbf{H})$ of culture in M16 (I) and CZBG(II) media. Photographed using phase contrast $(20 \times)$ objective. Magnification bars shown in panels $\mathrm{C}$ (identical in panels $\mathrm{A}-\mathrm{C}$ ) and $\mathrm{H}$ (identical in panels $\mathrm{D}-\mathrm{H})$ correspond to $50 \mu \mathrm{m}$.

injected embryos develop to blastocysts; similarly, with noninjected embryos (Table 1; Fig. 1). These observations are consistent with earlier published data (Chatot et al., 1994). Interestingly, the MCN of CZBG supportedblastocysts, that we observed (Table 2), is better than that reported earlier (Chatot et al., 1994). This might be due to the difference in glucose concentration (5.6 vs. $27 \mathrm{mM}$ ) and/or to the difference in the strain of mice used in the study.

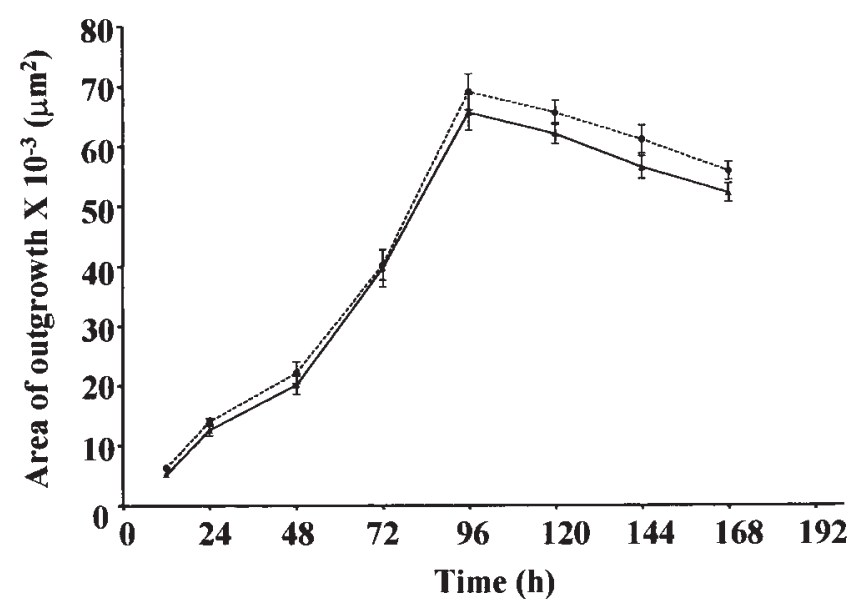

Fig. 6. Influence of M16 (-.-.) or CZBG $(-)$ media on trophoblast outgrowth of injected-mouse embryos through periattachment stages. Data are normalized to the time of hatching which corresponds to $0 \mathrm{hr}$ on the X-axis. Each value represents mean $\pm \mathrm{SEM}$ from 14 and 12 embryos, cultured in M16 and CZBG media, respectively. The observed decrease in values after $96 \mathrm{hr}$ was due to peeling off of trophoblast cells of the attached embryos in both treatments.
Our findings indicate that although supplementation of glucose at $48 \mathrm{hr}$ of culture (CZBG medium) is sufficient for morula to blastocyst transition, the presence of glucose from the beginning of culture (as in M16 medium) markedly enhance the number and quality of blastocysts developed from the injected embryos. This is consistent with an earlier observation where glucose is not shown to be an inhibitor of early embryo development when added at a concentration of $5.6 \mathrm{mM}$ in KSOM medium (Biggers and McGinnis, 2001). Similarly, the percentage development of blastocysts, cultured in M16 medium, and their MCN (noninjected) are consistent with earlier observation made with $\mathrm{F} 1$ hybrid CBA/C X C57BL/6 mice (Martin and Leese, 1995). However, M16 medium was shown to be inhibiting the development of out-bred mouse CF1 zygotes (Lawitts and Biggers, 1991). This variable effect of M16 medium may be due to differences in the strains of mice used for the study. In this regard, $\mathrm{FVB} / \mathrm{N}$ mice, that we employed, are one of the most common in-bred strains of mice for generating transgenic animals and subsequent genetic/breeding analysis (Taketo et al., 1991).

Incidentally, we observed that a 15 minutes exposure of pronuclear-stage embryos to M2 medium (contains $5.6 \mathrm{mM}$ glucose) is sufficient to promote their development to blastocysts (32\% noninjected and $13 \%$ injected), when cultured subsequently in CZB medium. The ability of glucose supporting morula-to-blastocyst transition, when embryos are exposed briefly to glucose at 1-cell-stage, can be explained based on early embryonic capability for glycogen synthesis and storage (Martin and Leese, 1995). Therefore, breakdown of 
I


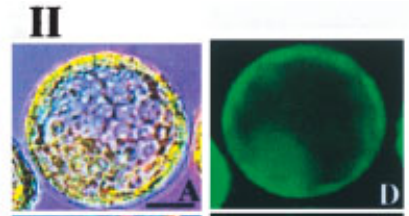

III
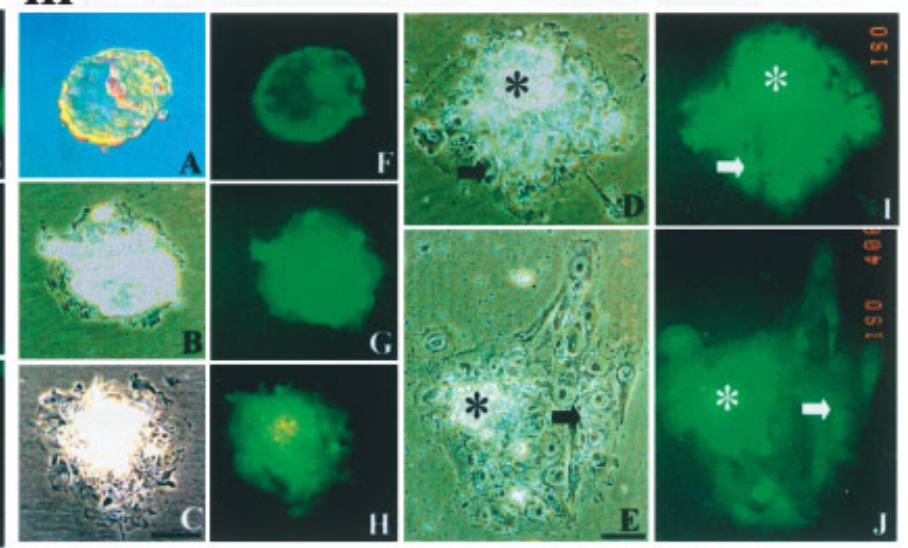

Fig. 7. In vitro development of EGFP-expressing mouse embryos through peri-attachment stages cultured in M16 medium. Panel I shows confocal optical sections of the EGFP-expressing blastocyst. Shown here are six representative images out of 16 sections, taken at various depths showing EGFP expression in trophectoderm ( $\Rightarrow$ ) and inner cell mass (). Panel II shows photomicrographs of expanded (A, D), hatching (B, E), and hatched $(\mathbf{C}, \mathbf{F})$ blastocysts. Panels A-C and D$\mathrm{E}$ correspond to DIC and their corresponding fluorescence pictures, respectively. Panel III shows progressive changes in the morphology

stored glycogen may provide glucose for morula-toblastocyst transition (Hsieh et al., 1979). Unlike in the mouse, observations made in other species, however, show that the presence of glucose is detrimental to development of early cleavage-stage embryos (Schini and Bavister, 1988; Seshagiri and Bavister, 1989, 1991; Petters et al., 1990; Takahashi and First, 1992; Thompson et al., 1992; Conaghan et al., 1993). This

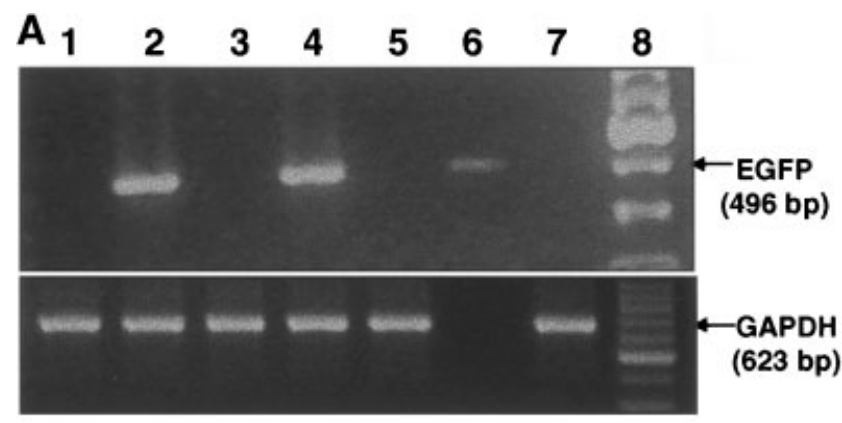

\section{B}

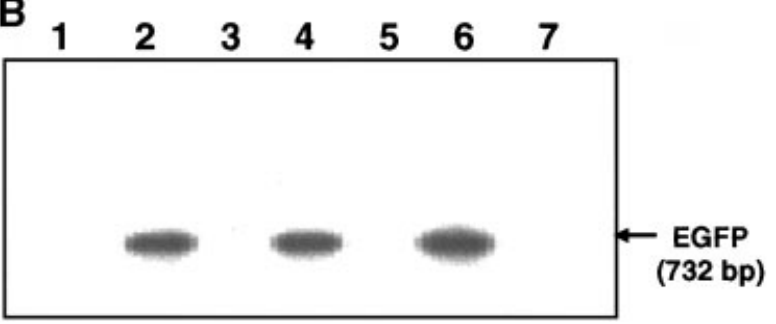

Fig. 8. Genotypic analysis of pups, born out of EGFP-injected cultured mouse embryos, by PCR (A) and genomic Southern (B) blot analyses. In both panels, lanes 1-5 correspond to tail DNA samples isolated from pups UT7, UT8, UT13, UT14, and UT19, respectively, derived from cultured blastocysts, following uterine transfer as detailed in text. Lanes 6-8 correspond to EGFP cDNA, control DNA and DNA marker (100 bp ladder plus), respectively.

of EGFP-expressing attached embryos, exhibiting fluorescence in trophoblasts $(\longrightarrow)$ and inner stem cells $(\boldsymbol{*})$, after $12(\mathbf{A}, \mathbf{F}), 24(\mathbf{B}$, G), $48(\mathbf{C}, \mathbf{H}), 72(\mathbf{D}, \mathbf{I})$, and $96(\mathbf{E}, \mathbf{J})$ hr in culture. Panels A-E and F-J correspond to DIC and their corresponding fluorescence images, respectively. Photographed using $20 \times$ objective. Magnification bars correspond to $50 \mu \mathrm{m}$ and are shown in panels I-F (identical in all panels of I), II-A (identical in panels C-D and F of II), II-B (identical in panel $\mathrm{E}$ of II), III-C (identical in panels $\mathrm{A}-\mathrm{C}$ and $\mathrm{F}-\mathrm{H}$ of III) and III-E (identical in panels D-E and I-J of III).

puzzling difference between the mouse on one hand and a variety of species on the other hand is quite intriguing and requires detailed comparative studies.

Although the components present in M16 and CZBG media are identical, except that the latter contains high lactate to pyruvate ratio and $1 \mathrm{mM}$ glutamine, the development of blastocysts, supported by M16 is better and their quality superior. This could be attributable to the two variabilities indicated above coupled with continues exposure of embryos to glucose from the beginning of culture in M16, unlike in CZBG media. Interestingly, the area of TB outgrowth of attached blastocysts (from injected embryos), cultured in either M16 or CZBG media, was not significantly different (Fig. 7). We believe that similar outgrowth patterns of blastocysts achieved in both media could be due to their comparable number of TE cells (Table 2).

We also observed relatively lesser percentages of blastocyst development and poorer quality of developed blastocysts of the EGFP-transgene injected embryos when compared with the noninjected embryos (Tables 1,2; Figs. 1,2). It could be argued that the microinjection-induced mechanical trauma of the former group may have resulted in the poor blastocyst development. We have discounted this possibility based on our observation that when $\mathrm{T}_{10} \mathrm{E}_{0.1}$ buffer-injectedeggs $(\mathrm{n}=163)$ were cultured in M16 medium, 81.3\% $( \pm 2.3)$ developed to blastocysts. Hence, we believe that the mechanical damage may not be the only factor for comparatively reduced blastocyst development of EGFP-injected embryos. We also considered the possibility that hyper-expression of EGFP during the preimplantation period may affect blastocyst development due to possible EGFP embryo toxicity. Though expression level-dependent inhibition of cell function and development may occur (Huang et al., 2000), we have 
evidence to show that developed blastocysts, which express low-moderate levels of EGFP, are developmentally competent, biological viable and produce live "green" mouse transgenic lines (see Results; Seshagiri and Devgan, 2002; Seshagiri et al., 2002). In this regard, it may be necessary to use M16 medium in embryoculture-based transgenesis approach to screen/select developmentally normal EGFP-expressing transgenic embryos (blastocysts) prior to embryo transfer, thereby improving percentage EGFP-transgenic offspring.

In conclusion, our results show that M16 medium appears to be better than the CZBG medium, so far as the in vitro pre- and peri-implantation development of (EGFP) transgene-injected embryos is concerned. We envisage that M16 medium could be quite advantageous to study the developmental effects of transgeneexpression (Yueh et al., 2000) and/or its behavior during pre- and peri-implantation embryo development (Gutierrez-Adan and Pintado, 2000). Importantly, M16 supported-blastocysts, developed from injected embryos, produce live offspring. Thus, M16 medium can be used to further improve the efficiency of embryoculture-based transgenesis (ECBT) in the mouse.

\section{ACKNOWLEDGMENTS}

The authors thank Prof. M. R. S. Rao for advise; Ms. M. Sarkar, S. Nyati, and Dr. Uday kumar for excellent technical support; Dr. Satish Totey for advice and help; the staff of Central Animal Facility for providing mice; and Ms. M.S. Padmavathy for help in the preparation of the manuscript.

\section{REFERENCES}

Ain R, Seshagiri PB. 1997. Succinate and malate improve development of hamster eight-cell embryos in vitro: Confirmation of viability by embryo transfer. Mol Reprod Dev 47:440-447.

Bavister BD. 1995. Culture of preimplantation embryos: Facts and artifacts. Hum Reprod 1:91-148.

Biggers JD, McGinnis LK. 2001. Evidence that glucose is not always an inhibitor of mouse preimplantation development in vitro. Hum Reprod 16:153-163.

Biggers JD, Whittingham DG, Donahue RP. 1967. Pattern of energy metabolism in the mouse oocyte and zygote. Proc Natl Acad Sci USA 58:560-567.

Chatot CL, Ziomek CA, Bavister BD, Lewis JL, Torres I. 1989. An improved culture medium supports development of random-bred 1-cell mouse embryos in vitro. J Reprod Fertil 86:679-688.

Chatot CL, Lewis-Williams J, Torres I, Ziomek CA. 1994. One-minute exposure of 4-cell mouse embryos to glucose overcomes morula block in CZB medium. Mol Reprod Dev 37:407-412.

Conaghan J, Hardyside AH, Winston RML, Leese HJ. 1993. Effect of pyruvate and glucose on the development of human preimplantation embryos in vitro. J Reprod Fertil 99:87-95.

Devgan V, Thomas M, Ullas KS, Rao MRS, Seshagiri PB. 2003. Embryo-culture-based generation of enhanced green fluorescent protein-transgenic mice. Biochem Biophys Res Commun 303:9941001.

Gardner DK. 1998. Changes in requirements and utilization of nutrients during mammalian preimplantation embryo development and their significance in embryo culture. Theriogenology 49: $83-102$.

Gutierrez-Adan A, Pintado B. 2000. Effect of flanking matrix attachment regions on the expression of microinjected transgenes during preimplantation development of mouse embryos. Transgenic Res 9:81-89.
Handyside AH, Hunter S. 1984. A rapid procedure for visualizing the inner cell mass and trophectoderm nuclei of mouse blastocysts in situ using polynucleotide-specific fluorochromes. J Exp Zool 231:429434 .

Hogan B, Costantini F, Lacy E. 1986. Manipulating the Mouse Embryo: A Laboratory Manual. Cold spring Harbor, NY: Cold Spring Harbor Laboratory press. pp 174-175.

Hsieh B, Chi MM-Y, Knor J, Lowry OH. 1979. Enzymes of glycogen metabolism and related metabolites in preimplantation mouse embryos. Dev Biol 72:342-349.

Huang WY, Aramburu J, Douglas PS, Izumo S. 2000. Transgenic expression of green fluorescence protein can cause dilated cardiomyopathy. Nat Med 6:482-483.

Kato M, Yamanouchi K, Ikawa M, Okabe M, Naito K, Tojo H. 1999. Efficient selection of transgenic mouse embryos using EGFP as a marker gene. Mol Reprod Dev 54:43-48.

Keiser JT, Jobst PM, Garst AS, Boone JT, Geyer CB, Phelps C, Ayares DL, Page RL. 2001. Preimplantation screening for transgenesis using an embryonic specific promoter and green fluorescent protein. Cloning 3:23-30.

Lawitts JA, Biggers JD. 1991. Overcoming the 2-cell block by modifying standard components in a mouse embryo culture medium. Bio Reprod 45:245-251.

Leppens-Luisier G, Sakkas D. 1997. Development, glycolytic activity, and viability of preimplantation mouse embryos subjected to different periods of glucose starvation. Biol Reprod 56:589-596.

Martin KL, Leese HJ. 1995. Role of glucose in mouse preimplantation embryo development. Mol Reprod Dev 40:436-443.

Mishra A, Seshagiri PB. 1998. Successful development in vitro of hamster 8-cell embryos to 'zona-escaped' and attached blastocysts: Assessment of quality and trophoblast outgrowth. Reprod Fertil Dev 10:413-420.

Petters RM, Johnson BH, Reed ML, Archibong AE. 1990. Glucose, glutamine and inorganic phosphate in early development of pig embryo in vitro. J Reprod Fertil 89:269-275.

Schini SA, Bavister BD. 1988. Two-cell block to development of cultured hamster embryos is caused by phosphate and glucose. Biol Reprod 39:1183-1192.

Seshagiri PB, Bavister BD. 1989. Glucose inhibits development of hamster 8-cell embryos in vitro. Biol Reprod 40:599-606.

Seshagiri PB, Bavister BD. 1991. Glucose and phosphate inhibit respiration and oxidative metabolism in cultured hamster eight-cell embryos: Evidence for the "Crabtree effect." Mol Reprod Dev 30:105111.

Seshagiri PB, Devgan V. 2002. Successful development of viable blastocysts from enhanced green fluorescent protein (EGFP) transgene-microinjected mouse embryos: Comparison of culture media. Biol Reprod 66(Suppl 1):155.

Seshagiri PB, Devgan V, Rao MRS. 2002. Embryonic expression of enhanced green fluorescent protein: Impact on development and transgenesis in the mouse; In: The proceedings of Serono International conference on Embryo Implantation: Cellular Changes from Bench to Bedside, CT, USA, pp 74.

Takada T, Iida K, Awaji T, Itoh K, Takahashi R, Shibui A, Yoshida K, Sugano S, Tsujimoto G. 1997. Selective production of transgenic mice using green fluorescent protein as a marker. Nat Biotechnol 15:458-461.

Takahashi Y, First NL. 1992. In vitro development of bovine one-cell embryos: Influence of glucose, lactate, pyruvate, amino acids and vitamins. Theriogenology 37:963-978.

Taketo M, Schroeder AC, Mobraaten LE, Gunning KB, Hanten G, Fox RR, Roderick TH, Stewart CL, Lilly F, Hansen CT, Overbeek PA. 1991. FVB/N: An inbred mouse strain preferable for transgenic analyses. Proc Natl Acad Sci USA 88:2065-2069.

Thompson JG, Simpson AC, Pugh PA, Tervit HR. 1992. Requirement for glucose during in vitro culture of sheep preimplantation embryos. Mol Reprod Dev 31:253-257.

Whittingham DG. 1971. Culture of mouse ova. J Reprod Fertil Suppl 14:7-21.

Yueh YG, Yaworsky PJ, Kappen C. 2000. Herpes simplex virus transcriptional activator VP16 is detrimental to preimplantation development in mice. Mol Reprod Dev 55:37-46. 